\title{
LA NUEVA FACULTAD DE BIOLOGÍA: EVALUACIÓN Y PERSPECTIVAS PROFESIONALES
}

\section{THE NEW FACULTY OF BIOLOGY: ASSESSMENT AND PROSPECTS}

\author{
AUTORES \\ Nicolás Jouve de la Barreda: Catedrático del área de Genética. Universidad de \\ Alcalá \\ nicolas.jouve@arrakis.es
}

\section{CURRÍCULUM VITAE}

Natural de Madrid. Catedrático de Genética de la desde 1977. Doctor en Ciencias Biológicas. Fue Presidente de la Sociedad Española de Genética (1900 a 1994). Imparte doctrina en la Universidad de Alcalá de Henares.

\section{RESUMEN}

Como probablemente conocen los lectores, la Facultad de Ciencias de la UAH ha sido escindida en dos Facultades, correspondientes a las dos Secciones de Biología y Química, que a partir de ahora continuarán organizando y planificando sus específicas titulaciones con independencia la una de la otra. Esto es algo que, movidos por razones de identidad profesional, muchos biólogos veníamos solicitando desde nuestra llegada a la Universidad de Alcalá, y que recomendaba el Comité de Evaluación Externa de la titulación de Biología, que emitió un detallado informe hecho publico a mediados del curso 1997-98. 


\section{PALABRAS CLAVE}

Facultad - Biología - Perspectivas profesionales

\section{ABSTRACT}

As you probably know the readers, the Faculty of Sciences of the UAH has been split into two faculties, for the two sections of Biology and Chemistry, which will now continue to organize and plan their specific qualifications independently of each other. This is something that moved for reasons of professional identity, many biologists we were requesting from our arrival at the University of Alcalá, and as recommended by the Committee for External Review of Biology degree, which issued a detailed report made public in mid-course 1997-98.

\section{KEY WORDS}

Faculty - Biology - Career Management

\section{ÍNDICE}

\section{Introducción}

2. ¿Existe un sentido de identidad profesional en Biología?

3. El Informe de Autoevaluación ¿para qué?

4. Aspectos diferenciales y posibles causas de la situación

5. Un buen momento para la Biología

6. La necesaria reforma del Plan de Estudios

7. ¿Y ahora qué? 


\section{Introducción}

Como probablemente conocen los lectores, la Facultad de Ciencias de la UAH ha sido escindida en dos Facultades, correspondientes a las dos Secciones de Biología y Química, que a partir de ahora continuarán organizando y planificando sus específicas titulaciones con independencia la una de la otra. Esto es algo que, movidos por razones de identidad profesional, muchos biólogos veníamos solicitando desde nuestra llegada a la Universidad de Alcalá, y que recomendaba el Comité de Evaluación Externa de la titulación de Biología, que emitió un detallado informe hecho publico a mediados del curso 1997-98.

Creo que quien lea estas líneas estará de acuerdo conmigo en que la Biología es una carrera vocacional y que quienes la eligen lo hacen principalmente movidos por un deseo de profundizar en el conocimiento de la naturaleza y del fenómeno de la vida. De acuerdo con una encuesta Socio-profesional de los Biólogos Españoles, realizada por el C.O.B. (Colegio Oficial de Biólogos) en 1994, entre 533 titulados la vocación es la principal motivación que impulsa a los jóvenes a matricularse en esta carrera. Pero además, se trata de una carrera con una vertiente aplicada de gran utilidad para diversos sectores socio-económicos, que emana del aprovechamiento de los recursos biológicos y del conocimiento de los procesos biológicos a todos los niveles, poblacional, individual, celular y molecular. Hoy, aproximadamente el 75\% de los biólogos españoles encuentran trabajo estable, si bien no de forma tan inmediata como ocurre en otras carreras de larga tradición profesional, como las técnicas. La mayoría de los biólogos trabajan en el sector servicios, y muchos se dedican a la enseñanza. Cerca de las dos terceras partes de los biólogos trabajan en el sector público, en enseñanza o en centros de investigación básica o aplicada (universidades, CSIC, INIA, Centros de Investigación de Comunidades Autónomas, etc.), y el resto en el sector privado. Siendo éstas las salidas tradicionales de los biólogos, hay otros campos de actividad profesional que reciben una creciente incorporación de estos 
titulados en los últimos años, como los servicios sanitarios, la agroalimentación, la industria farmacéutica y el medio ambiente.

Como todos saben, la Facultad de Ciencias se constituyó con sus dos secciones casi al comienzo de la andadura de la nueva Universidad de Alcalá, y desde entonces ha tenido su sede en uno de los viejos edificios heredados del antiguo aeródromo militar, actual "campus". Desde su nacimiento en 1977 hasta finales del año pasado ha mantenido un aceptable nivel de organización académica, que sin embargo, y dada la heterogeneidad académica ha diluido el carácter propio de las dos licenciaturas básicas que le daban razón de ser. Se trataba de una Facultad que desde fuera hacia dentro, tenía el denominador común y preponderante de ser una Facultad de experimentales, a continuación de Ciencias y en una tercera aproximación de Biólogos y Químicos, pero también de Geólogos, Físicos y Matemáticos. Si bien las dos licenciaturas de Biológicas y Químicas debían imprimir carácter a la Facultad, las diferencias profesionales y estructurales eran tan manifiestas que la unión se quedaba en un plano administrativo y organizativo, y la Facultad de Ciencias en un mero instrumento de administración de titulaciones. De hecho, fue necesario crear dos secciones con sus Vicedecanos y sus Juntas independientes para dar cauce a los temas específicos con la profundidad necesaria, lo que era imposible en un foro común por el lógico desinterés recíproco. Sin embargo, las Juntas de Sección carecían de poder de decisión, y al final cualquier tema específico debía ser elevado a la Junta de Facultad, con el correspondiente riesgo de su dilución, rechazo por falta de consenso o desinterés por más de la mitad de la Junta. La propia heterogeneidad, y la fuerte imbricación de los Departamentos generales, además de los de otras titulaciones con competencias asistenciales en Biología, contribuían a difuminar el sentido de identidad propio, probablemente de forma más acusada en Biología que en Químicas, por el mayor número y diversidad de sus áreas y la complejidad de su planificación docente. 


\section{2. ¿Existe un sentido de identidad profesional en Biología?}

La respuesta inmediata a esta cuestión, a la vista de los avances científicos en materias biológicas y sus enormes repercusiones socioeconómicas es que sí, aunque no se haga notar en nuestra Universidad. En mi opinión, la vida de la anterior Facultad de Ciencias ha distado de ser un modelo universitario de intercambio y debate en lo concerniente a la profesionalidad de sus titulaciones. Prácticamente no se ha propiciado una atmósfera de diálogo ni de estímulo de las mutuas relaciones académicas y profesionales de los biólogos, acorde con los tiempos que corren. Por contra, la apariencia de falta de objetivos en común ha propiciado el aislacionismo. Con ser esto de por sí lamentable, no es menos cierto que hay poca correspondencia entre esa triste imagen que se atribuye a los biólogos de desunión, y el nivel general de la licenciatura en Alcalá, varias décimas por encima en las encuestas de opinión sobre la docencia respecto al global de la Universidad, y con Departamentos y áreas excelentes en investigación, y con la mayor concentración de premios anuales del consejo social de la Universidad de Alcalá a la docencia y a la investigación.

Sin embargo, la imagen que proyecta la Biología en nuestra Universidad es a juicio de muchos de división interna. A veces pienso que hay razones objetivas que justifican a quienes nos juzgan de esa manera, dada la falta de solidaridad o el desinterés por los temas de carácter común de algunos compañeros, que decepcionados por las dificultades de relación entre las áreas de nuestra sección, se encierran en sí mismos, o se proyectan hacia fuera de nuestra Universidad. En mi opinión los biólogos somos más víctimas que culpables de esta situación y a pesar del tiempo transcurrido todavía habría remedio, si existiese conciencia de la realidad actual, y sí acertásemos con el camino adecuado en la nueva situación. 
No creo que la falta de identidad profesional que ahora padecemos sea consecuencia de una inconsciencia general, ni de una especie de locura colectiva de los biólogos, empeñados en tirar por la borda una hipotética situación ventajosa de que hubiésemos disfrutado, en base a la preocupación profesional de nuestras autoridades académicas, o por la modernidad, comodidad y holgura de nuestra diseminada infraestructura. A veces me pregunto qué está pasando en nuestra Universidad, y en nuestra Facultad para dejar pasar tantas oportunidades de diálogo científico. Qué clase de pobreza intelectual nos domina para enquistarnos en nuestro limitado entorno, y no enriquecernos con el conocimiento de los compañeros que, teóricamente al menos comparten una parcela de nuestro mismo campo científico y se mueven en especialidades distintas y complementarias. Estoy de acuerdo con el dicho popular de que el saber no ocupa lugar, pero también con la interpretación de nuestro insigne sabio D. Santiago Ramón y Cajal, cuando corrige el dicho y afirma que "el saber no ocupa lugar pero sí ocupa tiempo", por lo que siempre será mejor que nos expliquen nuestras dudas o curiosidades aquellos que las estudian y las conocen en mayor profundidad sencillamente porque les son más próximas. Es impresionante la cantidad de avances científicos que a diario nos llegan por las noticias y reportajes de los medios de comunicación y la prensa internacional sobre temas de Biología. Sin embargo, en los tiempos pasados nos ha aquejado una especie de sordera estéril para nosotros y para nuestros estudiantes. Salvo las iniciativas personales o de Departamentos aislados, ningún eco en nuestra Facultad, de temas como la clonación de células humanas, la ingeniería genética, las aplicaciones biotecnológicas del cultivo in vitro, el debate sobre bioética, los avances en la lucha contra el SIDA, el genoma humano, el origen y la evolución humana, la terapia génica, la transgénesis, la erosión genética, el impacto ambiental, los avances en neurotransmisión, la muerte celular, etc. Estos temas, no han promovido seminarios de información o de debate interno. La revolución biológica, como enfáticamente la denominan algunos medios de comunicación, o la adaptación de las ciencias de la vida al mercado y la investigación básica o aplicada, son cosa de la prensa, que no 
merecieron ni un minuto de atención encauzada o dirigida en la ya extinta Facultad de Ciencias. Apenas alguna conferencia de interés general para todas las ramas de la Ciencia, en San Alberto, aprovechando la coyuntura de la fiesta de todos.

\section{El Informe de Autoevaluación ¿para qué?}

En mi opinión, la falta de comunicación entre los responsables de la licenciatura de Biología, es consecuencia de una serie de males que vienen de largo y que han impedido el desarrollo de un auténtico sentido de identidad.

Desde la perspectiva de la titulación de Biología, nunca contamos con un decano biólogo cuando éramos Facultad de Ciencias. Nadie se preocupó del acercamiento entre las distintas áreas, ni se estimularon los seminarios, cursos o conferencias interdisciplinares, y mucho menos aún en relación con las salidas profesionales o la implicación del biólogo en la sociedad, la economía, etc. Tenemos los departamentos físicamente más dispersos y separados de toda la Universidad de Alcalá, hasta en cuatro edificios. Los que no se encuentran en Farmacia o Medicina, lo están en los edificios más viejos del campus. Algunos Departamentos interfacultativos, no hay ni que preguntar a qué Facultad están más vinculados, ya que la presencia física en Medicina, Farmacia, y últimamente CC Ambientales, parece imprimir tal carácter, que lo de biológicas parece "secundario", cuando no "utilitario".

Muchas de las causas y de las posibles soluciones a los problemas de nuestra Facultad, en lo que atañe a la licenciatura de Biología, fueron señaladas en el detallado informe emitido por el Comité de Evaluación Externa de la Licenciatura en Diciembre de 1997. Se trata de un Informe de Autoevaluación, promovido desde el Rectorado de la Universidad de Alcalá, que a través del análisis de la Titulación de Licenciado en Biología, Plan Nuevo, instaurado en el curso 1992-93, participaba en la 
primera convocatoria del Plan Nacional de Evaluación de la Calidad de las Universidades. Más de dos años después, salvo en lo que atañe a la separación de las dos secciones en Facultades, nadie parece haber asumido la trascendencia del citado Informe.

El comité de Evaluación repite en su extenso dictamen de forma machacona las deficiencias del espíritu profesional de la licenciatura tal como está planteada en nuestra Universidad. Así a propósito de las Metas, objetivos y planificación de la titulación, señala: "Las metas aparecen en nuestra opinión vagamente definidas y referidas a aspectos generales de la profesión"... "Debería haberse considerado el perfil de la profesión, a través de las ... funciones profesionales que se habrán de desarrollar y los ámbitos de trabajo en que podrán desarrollar dichas funciones". Y en otro lugar: "En opinión del comité de Evaluación, sería necesaria una mayor orientación profesional a los alumnos antes, durante y tras su paso por la Facultad".

Entonces, cabe preguntarse ¿a qué se debe esta falta de iniciativas para una modernización de la vida académica de la Facultad en lo que depende de nosotros?, pero también en lo que depende del Rectorado, que en su día se echó las manos a la cabeza con el Plan Actual de la Titulación de Biología, e inspiró la realización del Informe de Evaluación. ¿Cuál es la razón de tanta resistencia por parte de algunos compañeros por emprender una reforma en la dirección apuntada por el informe de Evaluación?. ¿Para qué estimuló el Rectorado un gasto de medios y energías, en reuniones y en informes que una vez publicados parecen olvidarse?. Sí la radiografía que hizo el Comité de Evaluación creemos que obedecía a una necesidad y se ajusta a un buen análisis de la situación, y de él se concluyen una serie de propuestas de mejora ¿a qué estamos esperando para ponerlas en práctica?. 


\section{Aspectos diferenciales y posibles causas de la situación}

A la vista del informe se detectan una serie de aspectos diferenciales, no necesariamente todos negativos, pero sí desequilibrantes. El primero es la diversidad de implicación de las áreas en la licenciatura. Las hay desde estrictamente biológicas en docencia y experimentación, hasta relacionadas solo con la docencia, a través de la impartición de una materia, a veces no troncal. Las hay que por dotación de profesorado pueden hacer frente a una especialidad de segundo ciclo, otras que se diversifican en dos o más especialidades, o incluso en una. Hay áreas que tienen a su cargo 28 asignaturas (del total de 121 que figuran en el actual plan), mientras que otras apenas ofrecen una. Algunas asignaturas de una determinada especialidad de segundo ciclo reciben más alumnos por libre elección que por la especialidad en la que se oferta. Algunas especialidades tienen más asignaturas y profesores que alumnos. Existen áreas que dedican cerca de 3000 horas en el conjunto de su docencia en la titulación al año, mientras que otras apenas pasan de 100. Las hay con sede física en otras Facultades, y con líneas de investigación de ámbito profesional diferente al biológico, y las hay específicamente biológicas, y con sede en Ciencias. Hay departamentos interfacultativos más volcados en otras titulaciones que en Biología, y otros esencialmente biológicos. Como digo más arriba, creo que la heterogeneidad en sí misma no tiene por qué ser mala, siempre que el desequilibrio que genera obedezca a razones lógicas de contenidos académicos, y no a situaciones históricas, heredadas ó consecuencia de estrategias o luchas de intereses no académicos.

Pero además, el reglamento de la extinta Facultad de Ciencias, heredado por la nueva Facultad de Biología, admite por igual como miembros natos a los Directores de los Departamentos de todas las áreas que contribuyen de una $\mathrm{u}$ otra manera a la docencia de la licenciatura, que son unos 15. Esto sucede a diferencia de lo que ocurre en otras Facultades, y con independencia del grado de implicación docente, 
número de asignaturas, carácter de éstas, etc. Esto, entre otras consecuencias, ha hecho aflorar en las Juntas de Sección y Facultad, cierto enfrentamiento ajeno al carácter formativo de la titulación, y más próximo a los intereses de crecimiento de las áreas implicadas. En la representación que se le supone a todo miembro de la Junta, a veces parece que importa poco o nada el aspecto académico, y la aproximación profesional hacia la biología, lo que prima es el vínculo administrativo. Se llega incluso a la disputa de las mismas materias desde diferentes áreas y perspectivas académicas, algo que por otra parte no es nuevo en nuestra Universidad dada la equivocada cultura imperante, sobre lo que tenemos todo tipo de ejemplos en varias facultades. A más asignaturas, más carga docente, más profesorado y más poder. Nadie pone en duda el respeto a todas las opiniones, pero en materia de una titulación, y en algo tan especial y delicado como la elaboración o reforma de un plan de estudios, no está de más reivindicar la exigencia de respeto para quienes están más próximos a la titulación, y conocen más de cerca la profesión. Algunas áreas de carácter general o asistencial, con representantes en la Junta de Facultad de Biología así lo entienden y son un modelo de comportamiento, por desgracia no tan generalizado como sería de desear.

Otro hecho de repercusión en la licenciatura de Biología en Alcalá, lo ha supuesto la creación de la carrera de Ciencias Ambientales sin contar con edificios ni infraestructura, ni profesorado específico, ni la opinión de la Facultad de Ciencias, pero eso sí con la generosa aportación de ésta en todo, aun a costa de las expectativas profesionales de los biólogos en materias de medio ambiente. En esto también hay que indicar que algunas áreas biológicas han encontrado un filón para aumentar sus cargas docentes, lo que no es objetable en modo alguno, pero tampoco contribuye de forma constructiva en pro de una determinada identidad.

Toda esta situación que afecta a los biólogos, puede sentirse de muy diferente manera. Desde un sentimiento de pesar y frustración hasta la total indiferencia, lo 
que está lógicamente en correlación con el grado de aproximación vocacional y anímica de cada persona hacia la licenciatura.

\section{Un buen momento para la Biología}

En los últimos años, a pesar de la situación presente, se habían ido produciendo una serie de hechos que permitían abrigar una cierta ilusión de cambio que habría de cristalizar en la creación de la nueva Facultad de Biología. Se había iniciado el proceso de adaptación-reforma del Plan de Estudios. Se podía pensar en unas instalaciones o edificios comunes, a lo que quizás seamos más sensibles los que parecemos apátridas, y con un rectorado tan sensible al sector de la construcción. Se había elaborado un informe detallado de evaluación de la licenciatura por el ya citado Comité de Evaluación, y existían unos planes de mejora.

El 15 de julio de 1998 el Tribunal Supremo dictó una importantísima sentencia para los biólogos. En ella se fallaba en contra de los recursos presentados por médicos, farmacéuticos y colegios de doctores y licenciados en Filosofía y Letras, lo que suponía un avance en la consolidación de nuestras competencias profesionales, que podría facilitar el acceso de los biólogos a las especialidades sanitarias, industriales, medio ambientales, etc., para las que nuestros licenciados están perfectamente formados y científicamente preparados. Los decanos de los centros en los que se imparten estudios de biología, que forman parte del consejo español, elaboraron una declaración conjunta en Abril de 1998 en la que se analizaba la situación y las excelentes perspectivas profesionales de la biología en España. Si desean saber algo más sobre esto pueden consultar la página de Internet del Colegio Oficial de Biólogos de España (http:/ / www.cob.es), y en particular el Artículo 15 de sus estatutos. 
Por otra parte, el presidente del Colegio Oficial de Biólogos, Pere Camprubí, en su ensayo "La Profesión de Biólogo", destacaba una serie de aspectos de gran relevancia que han ido surgiendo y están contribuyendo al desarrollo de la profesión. En coincidencia con nuestro ilustre compañero, yo señalaría algunos de los que están cambiando la imagen del biólogo. Este no ha de ser visto ya sólo como un mero defensor o contemplador de la naturaleza, sino como un profesional de la investigación, con conocimientos para modificar y aprovechar los recursos de los seres vivos, con implicaciones en industria, sanidad y medio ambiente, y con otros elementos de innovación como las aplicaciones industriales de los derivados y procesos de transformación biológicos, la emergencia de la mejora genética en agricultura y ganadería, la biotecnología aplicada al mundo sanitario, industrial y agropecuario, etc. Todo esto está cambiando realmente el panorama de la Biología, y cada vez son más los biólogos implicados no sólo en la contemplación de la naturaleza (tradicionalmente llamados de "bota") o en la enseñanza (de "tiza"), sino también en empresas, centros de investigación públicos y privados, investigación científica básica o aplicada y hospitales (de "bata").

El Comité de Evaluación Externa de la licenciatura de Biológicas, detectó los problemas que aquejan a la licenciatura y los centró en una serie de puntos que desembocaban en una serie de recomendaciones. Una de ellas era la separación de la Facultad de Ciencias en una de Químicas y otra de Biológicas. Otra la separación en dos especialidades, de la que actualmente se denomina Ambiental y Sanitaria. Se trata de una especialidad del Plan Actual que no se sostiene ni gusta a los alumnos que la cursan por razones divididas al 50\%. En definitiva el Comité de Evaluación sugería una reforma con detenimiento del Plan de Estudios. De este modo a propósito de "El Programa de Formación" se señala: "La aplicación del Plan de Estudios, instaurado en el curso 1992-93, propuesto para esta titulación, está causando una serie de disfuncionalidades, en cuanto a la organización docente en el Centro, y a los rendimientos académicos de los alumnos. Se observa que las causas 
fundamentales se relacionan con el alto grado de optatividad ofertado y con la existencia de un excesivo número de asignaturas (algunos egresados confesaron la necesidad de matricularse en un curso de 20 asignaturas,... lo cual no es extraño sí se tiene en cuenta que existen asignaturas con cuatro créditos). Dichos problemas son patentes tanto para los colectivos de profesores como de alumnos, y han creado conciencia de la necesidad de un reajuste del Plan de Estudios, ampliando el mismo a cinco años, sin un aumento de carga docente ni del número de asignaturas. Sería recomendable además que al abordar la reestructuración del mismo se considerara también el cumplimiento adecuado de los objetivos formativos de la titulación, así como el concepto de formación integral, donde el alumno menos agobiado por una excesiva permanencia en el Centro, pudiese asistir a conferencias, conciertos, actividades deportivas, etc." Y en otros lugares se insiste:... " falta de coordinación entre las diferentes prácticas que lleva a situaciones como la de coincidir tres sesiones prácticas el mismo día y hora",.... "La descoordinación de prácticas".... "La falta de información sobre salidas profesionales y prácticas de empresa". " La excesiva carga docente que el alumno tiene que soportar a lo largo de la titulación", y "La excesiva permanencia en el centro (10 a 12 horas)"

\section{La necesaria reforma del Plan de Estudios}

Es claro que ante la perspectiva de la división de la Facultad, uno de los elementos clave determinante de la firmeza de la nueva Facultad de Biología, aunque no el único, debía ser el nuevo Plan de Estudios. Así lo entendimos la mayoría de los miembros que formábamos parte de la Comisión Académica que trabajó en la adaptación del Plan, y que se propuso reformarlo a la luz de la nueva situación. El trabajo fue intenso y se procuró que el proyecto final recogiese las propuestas lógicas presentadas por todas las áreas representadas en la comisión que de forma directa o indirecta eran todas. El Proyecto debía orientarse en la dirección de las capacitaciones 
y salidas profesionales de los biólogos del umbral del siglo XXI. Había que atender a las demandas de la sociedad, y la elección de especialidades y los datos de los 6 ó 7 últimos años desde la implantación del Nuevo Plan constituyen elementos muy a considerar a la hora de abordar la adaptación obligada por Real Decreto, y la reforma recomendada por el Informe de Autoevaluación.

El Proyecto de adaptación-reforma estaba culminado a finales del curso 1998-99, y de hecho fue aprobado en una Junta de Sección el 20 de Septiembre de 1999, con un margen suficiente de votos y la posibilidad de introducir enmiendas por parte de aquellos que no lo aprobaban. Téngase muy en cuenta que el Proyecto llevaba el apoyo de 6 de las 7 áreas de mayor implicación en la carrera: Biología Animal, Biología Celular, Bioquímica y Biología Molecular, Ecología, Genética y Microbiología, y sólo se desvinculó el área de Biología Vegetal, a la que se adhirieron inmediatamente otras áreas de menor implicación docente, y más próximas a Medicina y Farmacia. Los motivos por los que estas áreas discrepaban pueden consultarse en el Acta de la sesión, y para no introducir mas elementos de discusión que los estrictamente necesarios, se resumen diciendo que hubo una diversidad de objeciones, incluso contradictorias entre sí, aunque una coincidencia de todas ellas en su acostumbrada visión "utilitaria" de la carrera. El argumento "estrella" era (es) la fijación en la pretendida pérdida o ganancia de créditos de unas áreas frente a otras. Consecuencia lógica de la cultura imperante, la supeditación de lo académico a la conveniencia. Peor aún, hay quien ha tratado de disimular las razones de carácter práctico para determinadas áreas (cambio de una especialidad por otras de corte más moderno o profesional) con argumentos de una pretendida conducta inmoral por parte de los miembros de la comisión, en el sentido de "barrer para sus áreas". En otras palabras, una buena defensa es un buen ataque, aunque objetívamente no se tenga razón. También se hicieron acusaciones infundadas de falta de visión del momento actual de la Biología a los miembros de la Comisión Académica, además de tildarles de obsoletos de principios de siglo (del XX claro) y otras lindezas, que no 
son sino la tinta del calamar, para ocultar una defensa a ultranza de otros intereses, que a ellos mismos deben parecer lo suficientemente vergonzosos como para defenderlos.

Un foco de discrepancia ha partido del Departamento de Biología Vegetal, constituido por dos áreas potenciales: Botánica y Fisiología Vegetal. Quien suscribe defendió en su día la existencia de las especialidades de Biología Vegetal y Biología Animal para la carrera. La práctica de los años de implantación del Plan Actual, desde 1992 han demostrado que aunque la primera es una especialidad muy atractiva, ha adolecido del éxito apetecido por todos, y apenas ha contado con el 7,2 $\%$ de los alumnos (unos 50 alumnos de cerca de 600 egresados desde la implantación del Plan Actual). La conveniencia de limitar a cuatro las especialidades, dividiendo la Ambiental-Sanitaria en dos, condujo a la comisión a una remodelación de las mismas, proponiendo la conservación de la que mayor éxito había tenido: Biología Celular y Molecular, que pasaría a denominarse Biología Molecular y Biotecnología, y daría cabida a nuevos contenidos si se juzgaba conveniente la inclusión de materias de las especialidades que desapareciesen. Además se recreaban dos especialidades una de Biología Sanitaria, y otra de Conservación, que junto con una cuarta de Evolución y Biodiversidad darían cabida a la oferta actual adaptada de las especialidades Vegetal y Animal. Todo ello a coste cero, sin pérdida de carga docente, y dando solución al error cometido en el actual plan, del reparto indebido por especialidades de las materias troncales de Fundamentos de Biología Aplicada, que deben ser comunes a todos los alumnos en el segundo ciclo. Esta sola modificación, y el cambio de las especialidades propuesta en el Informe de Autoevaluación, supone más que una adaptación una reforma del Plan. Téngase presente además que el tema de las "cargas docentes" no fue tratado por la Comisión que elaboró el Proyecto de adaptación del Plan hasta que no fue concluido el Proyecto de nuevo plan en todos sus detalles de estructura y contenidos, de especialidades y asignaturas. 
Sin embargo, de lo que se trataba para los discrepantes, no era de modernizar o adaptar un Plan a las nuevas tendencias de la Biología, en beneficio de nuestros estudiantes y futuros profesionales, sino de hacer una adaptación a la propia conveniencia de los Departamentos. En este sentido se cuestionan las salidas profesionales propuestas en Sanidad, Biotecnología, Conservación y Biodiversidad, frente a las de Animal, Vegetal, Ambiental-Sanitaria y Celular-Molecular. Cabe la esperanza de constatar que una mayoría de la anterior Junta de Sección votó con criterios más profesionales. Sin embargo, parece que para esta situación tampoco valen las mayorías, y en una nota reciente del Vicerrectotado de Planes de Estudios se nos hace saber que es necesario que "todos los miembros de la Junta" estén de acuerdo, supongo que para facilitar las cosas.

A pesar de todo, con la oposición indicada y con una mayoría suficiente, el Plan fue aprobado por Junta de Sección de Biología (embrión de la futura Junta de Facultad pero aun dependiente de la de Ciencias) en Septiembre pasado. La Facultad de Ciencias que sólo tenía que oficializar el Plan, una vez resueltas todas las enmiendas planteadas, hizo una nueva demostración de inoperancia en los temas específicos de una de sus Secciones. A pesar de lo previsible de los problemas que se podrían generar para el nacimiento de la nueva Facultad, le dejó una herencia terrible. La nueva Facultad de Biología, supuestamente más técnica que la anterior, debía de buscar el "consenso" y en su día aprobar el plan. De esta forma, y a pesar de las promesas del anterior equipo decanal, el Plan llegó a estar en el Orden del día en las dos últimas Juntas de Facultad previas a la separación, pero se evitó su tratamiento en Junta.

Quien suscribe, y habrá quien si lo desea pueda ratificarlo, avisó en el decanato de las previsibles consecuencias que podría acarrear el abandono del Proyecto. De este modo, en vísperas de la última Junta de Facultad de Ciencias, rogó que se 
considerase con las enmiendas incorporadas tras la última Junta de Sección. A pesar de ello, se decidió no tratarlo y trasvasarlo a la nueva Facultad de Biología, en vísperas de las elecciones de los representantes a la nueva Junta y de la elección de Decano. Mis argumentos se resumen en los siguientes aspectos inútilmente premonitorios:

a) dado que otra Junta es la que habrá de decidir la aprobación del Plan, tanto por parte de los que están de acuerdo (6 de las 7 áreas biológicas) como por los que no lo están (1 área biológica y otras menos vinculadas a la Biología), habrá movilización e interés por pertenecer a la nueva Junta.

b) una composición "sesgada" o "interesada" de representantes en Junta de Facultad podría afectar a todos los demás temas de la vida académica de la Facultad: elección de Decano, composición de las comisiones, reforma del reglamento de la Facultad, etc.

c) el Plan, que tanto tiempo y esfuerzo había supuesto para los que con ilusión y esfuerzo habíamos trabajado en él, cerca de 2 años, 21 reuniones y muchas horas de estudio, entrevistas, consultas y conversaciones, no llegaría a tiempo para su homologación antes del 1 de octubre del año 2000. Este era el plazo preceptivo para las Universidades de acuerdo con el RD 779/1998, como explícitamente nos indicaban los "criterios generales de actuación para la adaptación de los Planes de Estudio en la Universidad de Alcalá" del Vicerrectorado de Ordenación Académica y Planes de Estudio.

Punto por punto, es lamentable constatar cómo de Enero a Abril se han cumplido los tres puntos anteriores. Aun peor, ha habido un boicot, por parte de unos estudiantes, de la Junta de Facultad en que había de tratarse el Proyecto con posibilidades de llegar a tiempo. 
En lo que antecede, he ido exponiendo algunos de los argumentos que, por encargo oficial de nuestro nuevo Decano, hubiera desarrollado en dicha Junta en defensa del Proyecto y de la Comisión Académica que lo elaboró. Con respeto a los que discrepan del Proyecto, pero con la firmeza de quienes han dedicado muchas horas de trabajo en él, debo decir que nunca en mi trayectoria de 35 años en 5 universidades he vivido un espectáculo tan bochornoso, antiacadémico y antidemocrático como el protagonizado por dichos estudiantes y sus inspiradores.

Preguntar a los estudiantes sobre lo que han perdido al boicotear una Junta en la que el Orden del día incluía la posible aprobación del Proyecto, es un ejercicio inútil. Ya se darán cuenta de su error, entre otras cosas cuando sigan bajo la presión de la carrera en 4 años, con más de 6 asignaturas simultáneas (y hasta 20 en algunos casos), una oferta ficticia de optativas en algunas especialidades, y un sin fin de problemas de coordinación, además de pagar más matrícula y más créditos en el curso que viene. Es inútil explicarles el daño que se han hecho a sí mismos (una minoría de irresponsables), o a sus compañeros (una mayoría de despistados), en sus posibles expectativas profesionales.

\section{7. ¿Y ahora qué?}

Los que hemos trabajado de forma aséptica en el Plan somos testigos de cómo se han movido una serie de elementos ajenos a los criterios académicos y profesionales. Ahora solo falta esperar a que la nueva Comisión Académica, presidida por el nuevo Decano, trabaje sobre el Proyecto elaborado por la Comisión anterior. Según el acuerdo adoptado en la Junta de Facultad del pasado 10 de Abril, la Junta de Facultad habrá de partir de dicho proyecto y pronunciarse sobre las decisiones importantes que deban tomarse al respecto. Nada que objetar a este sometimiento a 
la Junta sobre la marcha si surgiesen dificultades. Es algo que yo mismo sugerí en diversas ocasiones durante la elaboración del Proyecto. Hay muchos que esperamos y deseamos escuchar y discutir en el seno de la nueva Comisión, y en la Facultad si hiciese falta, los argumentos supuestamente académicos que justifican el no.

Vamos a ver sí la nueva junta de Facultad es realmente más técnica y académica que la anterior. Debe exigirse que haya más imaginación, y no simplemente una descalificación sin argumentos. O que éstos sean simplemente que lo que se propone "no me gusta", sin más explicaciones, en lugar de "no me conviene". Espero que la nueva situación no esté condicionada por unos prejuicios de lo bueno (o malo) para mí es bueno (o malo) para todos. Es difícil esperar milagros, sobre todo después de oír en una Junta reciente como se descalifica a quienes participaron en la elaboración del Proyecto de adaptación, y olvidando la dedicación, el trabajo, y todo lo demás, proponen empezar de nuevo, sólo porque eso fue otra Junta, y una etapa pasada no debe obligar a la nueva Facultad. Este tipo de declaraciones deja bien a las claras cuales son las intenciones de quien las pronuncia.

Hay otros aspectos, relacionados con todo lo anterior, que al que suscribe le plantean muchas dudas, y que afectan a "autoridades académicas". Si ha lugar, y en virtud de lo que vayamos viendo, hechos que no palabras, hablaremos. Pero aquí ya se sabe, hoy digo una cosa y mañana otra, y pasado otra distinta, y siempre tendré la razón, porque al final no importa lo que diga sino quien lo dice, a quien se lo dice, y que salga el sol por Nuevo Baztán, que es un pueblo muy bonito.

Nicolás Jouve de la Barreda es Biólogo, Catedrático de Genética, y Premiado por el Consejo Social de la Universidad de Alcalá en Investigación (1990) y Docencia (1996)

12 de Mayo de 2000 